(C) The Authors 2017. This is an Open Access article, distributed under the terms of the Creative

Commons Attribution licence (http://creativecommons.org/licenses/by/4.0/), which permits unrestricted

re-use, distribution, and reproduction in any medium, provided the original work is properly cited.

\title{
Prenatal exposure to vitamin D from fortified margarine and risk of fractures in late childhood: period and cohort results from 222000 subjects in the D-tect observational study
}

\author{
Mina Nicole Händel ${ }^{1,2 *}$, Peder Frederiksen ${ }^{2}$, Clive Osmond ${ }^{3}$, Cyrus Cooper ${ }^{3}$, Bo Abrahamsen ${ }^{1,4}$ and \\ Berit L. Heitmann ${ }^{2,5,6,7}$ \\ ${ }^{1}$ Department of Clinical Research, Odense Patient Data Explorative Network (OPEN), Odense University Hospital, University of \\ Southern Denmark, 5000 Odense C, Denmark \\ ${ }^{2}$ Research Unit for Dietary Studies, Bispebjerg and Frederiksberg Hospital, The Parker Institute and the Institute of Preventive \\ Medicine, 2000 Frederiksberg, Denmark \\ ${ }^{3}$ Medical Research Council Lifecourse Epidemiology Unit, University of Southampton, Southampton SO16 6YD, UK \\ ${ }^{4}$ Department of Medicine, Holbcek Hospital, DK-4300 Holboek, Denmark \\ ${ }^{5}$ Section for General Practice, Department of Public Health, Copenhagen University, Øster Farimagsgade 5, opg. Q, 1014, \\ Copenhagen K, Denmark \\ ${ }^{6}$ The Boden Institute, Charles Perkins Centre, University of Sydney, D17, Johns Hopkins Drive, Camperdown NSW 2006, \\ Sydney, Australia \\ ${ }^{7}$ National Institute of Public Health, University of Southern Denmark, Øster Farimagsgade 5A, 2. 1353 Copenhagen K, Denmark
}

(Submitted 11 August 2016 - Final revision received 16 January 2017 - Accepted 3 March 2017-First published online 10 April 2017)

\begin{abstract}
Prenatal low vitamin D may have consequences for bone health. By means of a nationwide mandatory vitamin D fortification programme, we examined the risk of fractures among 10-18-year-old children from proximate birth cohorts born around the date of the termination of the programme. For all subjects born in Denmark during 1983-1988, civil registration numbers were linked to the Danish National Patient Registry for incident and recurrent fractures occurring at ages 10-18 years. Multiplicative Poisson models were used to examine the association between birth cohort and fracture rates. The variation in fracture rates across birth cohorts was analysed by fitting an age-cohort model to the data. We addressed the potential modification of the effect of vitamin D availability by season of birth. The risk of fractures was increased among both girls and boys who were born before the vitamin D fortification terminated in 1985 (rate ratio (RR) exposed $v$. non-exposed girls: 1.15 (95\% CI 1.11, 1.20); RR exposed $v$. non-exposed boys: 1.11 (95\% CI 1.07, 1.14). However, these associations no longer persisted after including the period effects. There was no interaction between season of birth and vitamin D availability in relation to fracture risk. The study did not provide evidence that prenatal exposure to extra vitamin D from a mandatory fortification programme of $1.25 \mu \mathrm{g}$ vitamin $\mathrm{D} / 100 \mathrm{~g}$ margarine was sufficient to influence the risk of fractures in late childhood, regardless of season of birth. Replication studies are needed.
\end{abstract}

Key words: Epidemiology: Vitamin D: Fracture risk: Fortification: Children

Based on serum 25-hydroxy vitamin $\mathrm{D}_{3}\left(25(\mathrm{OH}) \mathrm{D}_{3}\right)$ measurements, studies have shown a pronounced seasonal variation in vitamin D status in Denmark and other countries with latitudes above $35^{\circ}$ North and South ${ }^{(1,2)}$, most likely related to insufficient actinic vitamin $\mathrm{D}$ synthesis during the darker part of the year in such countries. Consequently, the vitamin D status will then depend more on diet, supplementation and/or fortification alone. In Denmark, the reintroduction of fortified foods to improve the general population's vitamin D status is currently being considered, and its justification is part of an ongoing international debate.

Until 1 June 1985, vitamin D fortification of margarine was mandatory in Denmark, but fortification was abolished, related to an unsupported assumption that the amounts added to margarine were too small to impact the dietary needs of vitamin D in the Danish population ${ }^{(3)}$. We used this historical change in fortification legislation to examine the influence of extra prenatal vitamin $\mathrm{D}$ exposure from fortified margarine on

Abbreviations: $25(\mathrm{OH}) \mathrm{D}$, 25-hydroxy vitamin D, ICD, International Classification of Diseases.

*Corresponding author: M. N. Händel, email Mina.Nicole.Holmgaard.Handel@regionh.dk 
the risk of fractures during pubertal-related growth spurts, which has the highest fracture incidence ${ }^{(4)}$.

Vitamin D and its metabolites play essential roles in regulating $\mathrm{Ca}$ homoeostasis in the intestine, kidney and bone. The evidence for linking maternal vitamin D insufficiency to offspring fracture rates is sparse and is based on results from animal studies ${ }^{(5)}$, as well as observational studies suggesting that maternal vitamin D status influences fetal bone growth and mineralisation ${ }^{(6)}$, and perhaps also long-term bone health among offspring ${ }^{(7-12)}$. Given that low bone mineral density (BMD) is predictive of increased fracture risk, as shown in both case-control and prospective studies - though principally in adults $^{(13-20)}$ - maternal vitamin D insufficiency may potentially also influence fracture risk.

Among children and adolescents, the most common fracture site is the forearm ${ }^{(21-25)}$, followed by the carpal bones, clavicle and foot/ankle ${ }^{(26)}$. Across European countries, the seasonality of fractures exhibit notable similarities, with peaks during summer, with a notable drop in the month of July and a nadir during winter ${ }^{(4,22,24,25,27,28)}$.

We hypothesised that individuals born during the last 2 years of the mandatory vitamin D fortification had a reduced risk of sustaining fractures of the forearm, wrist or scaphoid bone, clavicle and ankle in late childhood, compared with those born 2 years after the termination of vitamin D fortification, allowing for a washout period after termination. In addition, it was hypothesised that the vitamin D fortification during sundeprived months of gestation would be associated with the greatest risk reduction of offspring childhood fractures.

\section{Methods}

\section{Study design}

The D-tect study design ${ }^{(29)}$ relies on a natural experiment, defined as an exposure to an event or intervention, which has not been manipulated by the researcher ${ }^{(30)}$. The D-tect study is based on the fact that until 1 June 1985 it was mandatory in Denmark to fortify all margarine with vitamin D. Margarine was fortified with $1.25 \mu \mathrm{g} / 100 \mathrm{~g}$ and approximately $13 \%$ (3-29\%) of all dietary vitamin $\mathrm{D}$ is estimated to have come from the fortified margarine ${ }^{(31)}$.
We did not identify other abrupt societal changes during 1983-1988 that potentially could influence our results, neither in relation to fortification practices in other food products for consumption $^{(31,32)}$, nor in relation to margarine intake in the Danish population ${ }^{(33)}$ or in relation to national recommendations for vitamin D supplementation to pregnant women or infants. Therefore, any confounding would be expected to have influenced the exposed and non-exposed individuals from the two groups similarly, making them fully comparable. Hence, prenatal exposure to extra vitamin $\mathrm{D}$ from fortification is assumed to be the only parameter that separates the individuals in the two exposure groups.

\section{Study population}

All individuals born alive in Denmark from 1 January 1983 to 31 December 1988 were included in the study. We divided individuals from the birth cohort into different exposure groups. The exposed individuals were defined with birth dates from 1 June 1983 to 31 May 1985, and the non-exposed individuals were those with birth dates from 1 September 1986 to 31 August 1988. Between the exposed and the non-exposed groups, we included a washout period ( 9 months of pregnancy plus 6 months of margarine shelf-life) in the time period from 1 June 1985 to 31 August 1986. In order to use the full potential of the data set available, we included a run-in period with individuals born from 1 January 1983 to 31 May 1983, and a late period running from 1 September 1988 to 31 December 1988 (Fig. 1). Hence, individuals born before the termination of the mandatory fortification were exposed prenatally, but not during childhood (the exposed group), and those born after the fortification termination were neither exposed prenatally nor during childhood (the non-exposed group).

Follow-up time for fractures for each participant started at age 10 years (or the age at 1 January 1996 if the participant at that date was older than 10 years) and ended at death, emigration, disappearance or age 18 years, whichever came first. An overview of the study population is presented in Table 1.

Using the civil registration numbers, each individual was linked to the Danish National Patient Registry (NPR) for incident and recurrent diseases. The register contains information about hospital contacts, including diagnosis codes and procedure

$$
\begin{gathered}
\text { Run-in } \\
\text { (Jan 1983-May 1983) }
\end{gathered}
$$$$
\text { Exposed }
$$

(June 1983-May 1985)

$$
\begin{gathered}
\text { Washout } \\
\text { (June 1985-Aug 1986) }
\end{gathered}
$$

Non-exposed (Sep 1986-Aug 1988)

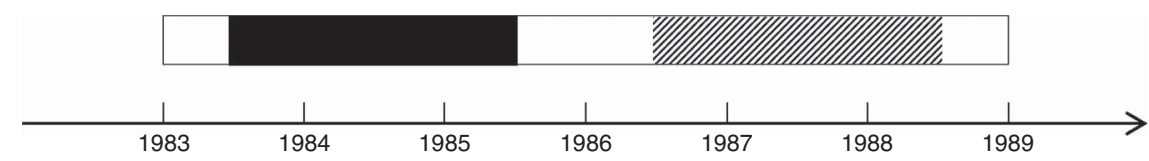

Fig. 1. Definition of the exposure groups. Vertical lines indicate the timing of the cohorts around the vitamin $D$ fortification termination date (31 May 1985 ). Exposed; $\mathbb{Z}$, non-exposed; $\square$, run-in, washout and late cohort. 
Table 1. Number of individuals contributing with risk time, person-years, and number of fracture events in the study population by birth cohort exposure groups and sex

\begin{tabular}{|c|c|c|c|c|c|c|}
\hline & Run-in & Exposed & Washout & Non-exposed & Late & Total \\
\hline Boys (total) & 10974 & 53555 & 35308 & 58542 & 9665 & 168044 \\
\hline Person-years & 54035 & 321870 & 263149 & 446284 & 74230 & 1159568 \\
\hline Fractures (total) & 1298 & 7636 & 5984 & 9623 & 1566 & 26107 \\
\hline Forearm, wrist or scaphoid bone & 950 & 5706 & 4521 & 7361 & 1221 & 19759 \\
\hline Ankle & 207 & 1101 & 830 & 1241 & 187 & 3566 \\
\hline Clavicle & 141 & 829 & 633 & 1021 & 158 & 2782 \\
\hline Girls (total) & 10458 & 50851 & 33580 & 55035 & 9286 & 159210 \\
\hline Person-years & 52875 & 313792 & 255056 & 426974 & 72442 & 1121138 \\
\hline Fractures, total & 673 & 4694 & 4063 & 6435 & 1051 & 16916 \\
\hline Forearm, wrist or scaphoid bone & 490 & 3609 & 3258 & 5108 & 878 & 13343 \\
\hline Ankle & 136 & 805 & 597 & 918 & 123 & 2579 \\
\hline Clavicle & 47 & 280 & 208 & 409 & 50 & 994 \\
\hline
\end{tabular}

codes for all treatments at Danish hospitals ${ }^{(34)}$. The main study outcome was fracture of the forearm, wrist or scaphoid bone (International Classification of Diseases (ICD)-10: S52, S62.0); fracture of the clavicle (ICD-10: S42.0); and fracture of the ankle (ICD-10: S82.5, S82.6, S82·8). From 1994, ICD-10 diagnoses were classified according to the WHO International Classification of Diseases, and from 1 January 1995 the outpatient and emergency room contacts were mandatorily included in the registers $^{(35)}$. Because of this incompleteness in the NPR, the individuals were considered at risk only from their age as on 1 January 1996 onwards. Recurrent fracture events were allowed, but if an individual had more than one fracture admission per event, we counted only the first admission and embedded a washout period of 6 months after a fracture at the same anatomical location, in order to avoid inflating the fracture risk estimates by including readmissions.

Risk time (person-years) and the number of fracture events were classified by sex, date of birth, age and calendar time during follow-up in monthly classes in a Lexis diagram ${ }^{(36)}$.

There are no ethical considerations regarding the epidemiological aspects of the study as only pre-existing databases and registries that had already been approved were used. According to Danish law, ethical approval is not required for purely registerbased studies. Permission for conducting the study was granted by the Danish Data Protection Agency (J. no.: 2012-41-1156).

\section{Statistical analysis}

Multiplicative Poisson models were used to examine the association between birth cohort and fracture rates. Rates were analysed by models for the number of fractures with the log-person-years as offset. With the Poisson model it is possible to explicitly model the underlying rates, and thus obtain estimates describing the fracture rates by age at occurrence, time of occurrence and time of birth (age, period, cohort). The analysis was performed for fractures overall and for fracture subtypes, separately for each sex. Power calculations for the present study have previously been published ${ }^{(29)}$. In brief, as the follow-up period varies, the final prevalence ( $=$ incidence $\times$ duration) was used, and it varied from 0.5 to $8 \%$. The significance level was $\alpha 0.05$ and power $\beta 0.80$. The least detectable relative risk of outcome after change in fortification (assuming that either one (at least 100000 individuals) or two birth cohorts are included) varied from 1.19 for a prevalence of $0.5 \%$ to 1.05 for a prevalence of $8 \%$.

First, we described the variation in facture rates across birth cohorts by fitting an age-cohort model to the data. In a second series of models, the birth cohorts were divided into the five exposure groups mentioned above (run-in, exposed, washout, non-exposed, late period). In a third series of models, we addressed the potential modification of the effect of vitamin D availability by season of birth, with winter defined as births in November, December or January. We tested for interaction between season of birth and exposure in relation to fracture risk by likelihood ratio tests. In a fourth series of models, we included period effects. First, we fitted an age-period model to the data (with July 2001 as the reference period) and subsequently, a cohort-only model to the residuals (i.e. using the fitted values from the age-period model as offset). The estimated cohort effect is then the ratio between the observed number of fractures within the cohort and the expected number of fractures, where the expected number is based on the predicted rates from the age-period model. This sequential approach to age-period-cohort modelling corresponds to fixing the cohort effects to have no overall trend ${ }^{(36)}$.

All data management was performed in Stata version 13.1, and statistical analyses were performed in R (R Foundation for Statistical Computing).

\section{Results}

In total, 327254 children contributed with risk time during ages 10-18 years. We identified 104406 individuals in the exposure period, of which approximately 51\% were boys, and 113577 individuals in the non-exposed period, of which approximately $52 \%$ were boys. In the run-in, washout and late periods, there were 21432, 68888 and 18951 individuals, respectively. In total, 12330 exposed and 16058 non-exposed individuals sustained a fracture with an overall fracture rate of 19.4 (95\% CI 19.1, 19.7) and 18.4 (95\% CI 18.1, 18.7) per 1000 person-years among the exposed and non-exposed individuals, respectively. The fracture type with the highest incidence was forearm, wrist or scaphoid bone, with 9315 events in the exposed group and 12469 events in the non-exposed group (Table 1). 

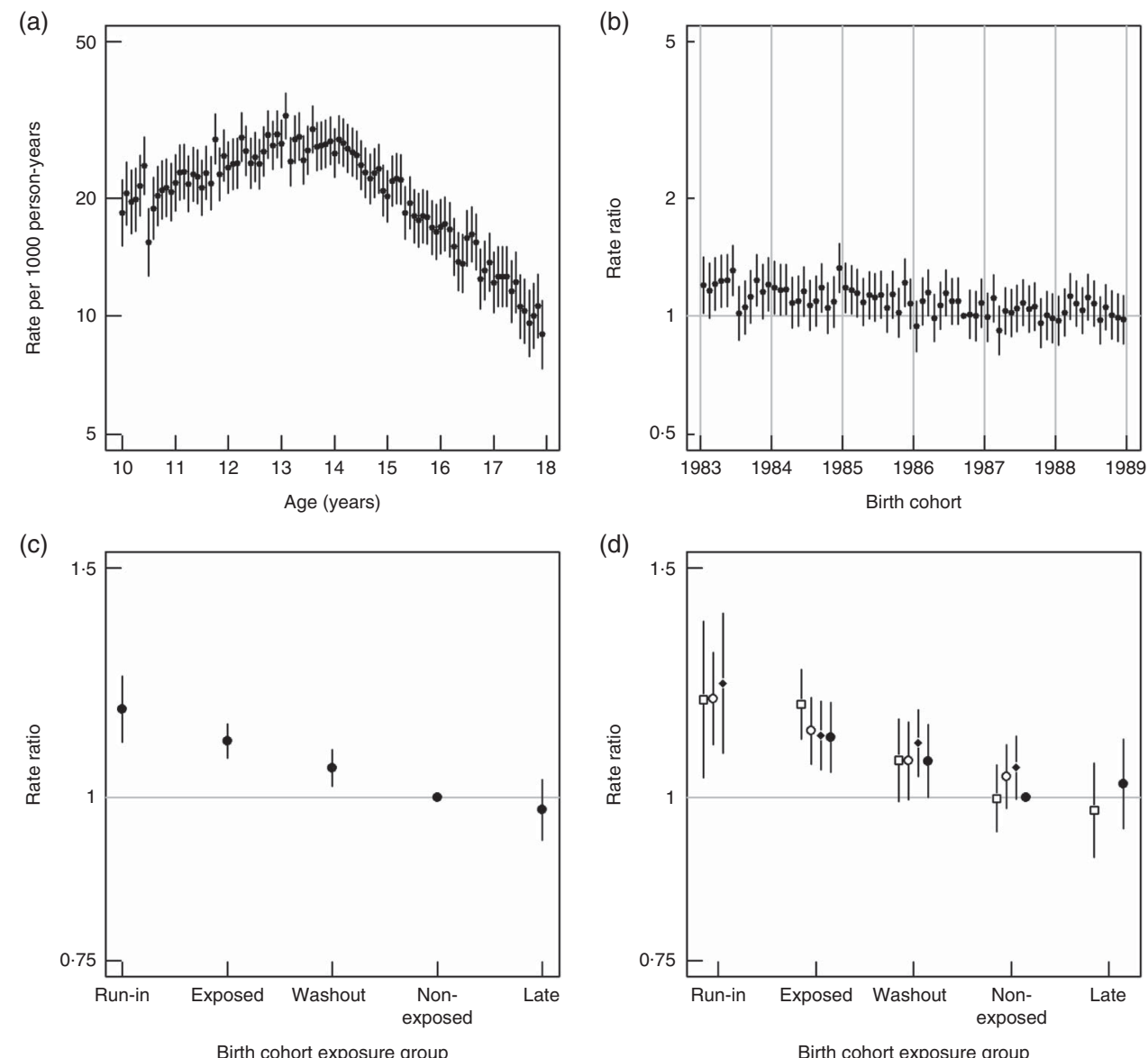

Fig. 2. Age and birth cohort effects for boys born in 1983-1988. (a) Age specific fracture rates per 1000 person-years and $95 \% \mathrm{Cl}$ for boys born in September 1986 , (b) rate ratio relative to September 1986 cohort, (c) rate ratios by birth cohort exposure groups ('Non-exposed' cohort is reference) and (d) rate ratios by birth cohort exposure group and season of birth (birth season 'August-October' and 'Non-exposed' cohort is reference). $\square$, November-January; $\bigcirc$, February-April; $\$$, May-July; - August-October.

\section{Fracture risk across age groups and seasonality in fracture occurrence}

As expected, the fracture rates differed according to age and there was a rate difference between boys and girls. Among the boys, the overall average fracture rate was $22 \cdot 5 / 1000$ personyears, with a peak fracture rate of $35 \cdot 1 / 1000$ person-years between the ages of 13 and 14 years (Fig. 2 and 3). Among the girls, the overall average fracture rate was $14 \cdot 6 / 1000$ personyears, with a peak fracture rate of 27.9/1000 person-years between the ages of 11 and 12 years (Fig. 4 and 5).

The peak age of the ankle and clavicle fracture rates occurred later compared with the overall fracture rate, but was similar for both girls (range of 12-14 years) and boys (range of 15-16 years) (online Supplementary Fig. S1-S8). The overall average fracture rate was $2 \cdot 7 / 1000$ person-years for ankle and $1 \cdot 7 / 1000$ personyears for clavicle, respectively. Forearm, wrist or scaphoid bone fractures showed a similar pattern as the overall analysis, resulting from the high incidence rate of forearm, wrist or scaphoid bone fractures in children, which was the main contribution to the fracture outcome (online Supplementary Fig. S9-S12).

The estimates from the age-period model revealed a seasonal fracture pattern with increased risk during spring and early fall and with a nadir during winter in the period from 1996 to 2007 (Fig. 2 and 5).

A summary of the month-by-month periodicity is presented in Table 2. For both girls and boys, we observed an almost 2-fold increase in the fracture rate when comparing the months with the highest rates (April, May or August) and the month with the lowest rate (December).

\section{Fracture rates compared between individuals potentially exposed to vitamin D fortification and} non-exposed individuals

Among girls, the rate ratio for the exposed was 1.15 (95\% CI $1 \cdot 11,1 \cdot 20)$ compared with the non-exposed. Among boys, the rate ratio for the exposed compared with the non-exposed was $1 \cdot 11$ (95\% CI 1.07, 1.14) (Fig. 2 and 5). However, these associations no longer persisted after including the period effects. The downward trend seen in the estimates from the age-cohort model has successfully been assigned as a period effect and there appears to be no systematic variation left in the cohort term based on the residuals. The relative risk for the exposed girls was 1.01 (95\% CI $0 \cdot 96,1.05)$ compared with the non-exposed girls, and the relative 

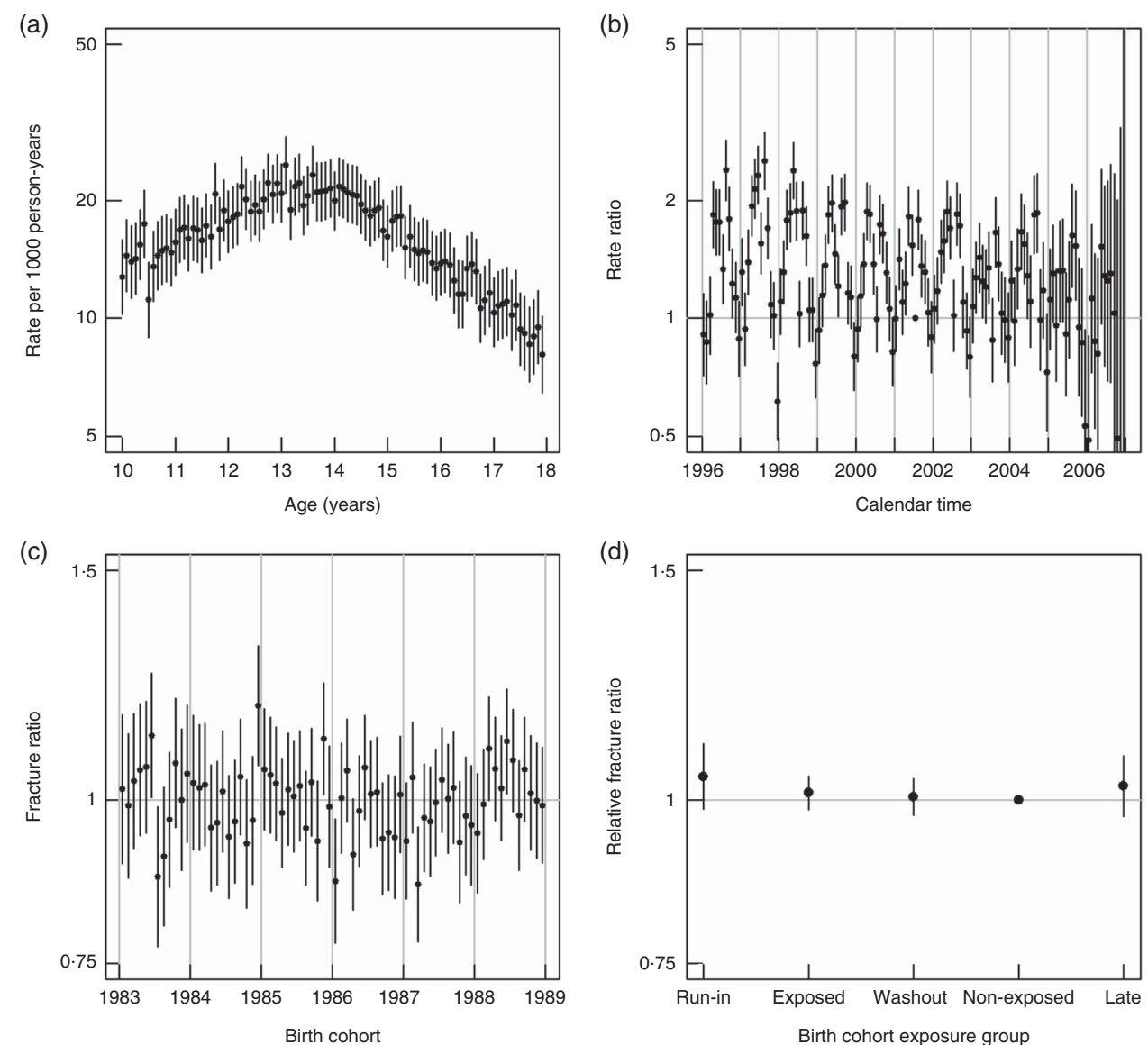

Fig. 3. Age and period effects for boys with fractures occurring from 1996-2007. (a) Age specific fracture rates per 1000 person-years and $95 \% \mathrm{Cl}$ for boys in June 2001, (b) rate relative to the July 2001 rate, (c) observed $v$. expected number of fractures conditional on the estimated age and period rates and (d) cohort effect by birth cohort exposure group relative to the cohort effect in the 'Non-exposed' cohort.

risk for exposed boys compared with the non-exposed boys was 1.01 (95\% CI 0.98, 1.04) (Fig. 2 and 5).

For both girls and boys, there was no interaction between season of birth and the exposure to vitamin $\mathrm{D}$ fortification in relation to overall fracture risk (girls: $P$ 0.23; boys: $P$ 0.44).

\section{Discussion}

This study assessed the long-term risk of childhood fractures from the natural experiment of terminating a mandatory margarine fortification programme that until 1 June 1985 fortified all margarine with vitamin D in Denmark. The study did not provide evidence that prenatal exposure to extra vitamin $\mathrm{D}$ from fortification was sufficient to influence the risk of fractures in late childhood, regardless of season of birth.

Interestingly, the fortification programme may have taken place against a long-term trend of a decreasing fracture risk, including these particular birth cohorts under study. Thus, we cannot rule out whether the birth cohort results reflect a true adverse effect of vitamin D on bone; one previous study did indeed report borderline significant inverse associations between maternal late pregnancy $25(\mathrm{OH}) \mathrm{D}$ concentrations and offspring forearm fractures ${ }^{(12)}$. Also, in the Danish National Birth Cohort, mid-pregnancy maternal supplementation with
$>10 \mu \mathrm{g}$ vitamin $\mathrm{D} / \mathrm{d}$ was associated with a $30 \%$ higher risk of offspring forearm fractures ${ }^{(37)}$.

Nonetheless, the decreasing trend may have been affected by influences more powerful than the likely impact of vitamin $D$ in the modest amounts of $1.25 \mu \mathrm{g}$ vitamin $\mathrm{D} / 100 \mathrm{~g}$ margarine ${ }^{(31)}$. By comparing individuals from entire adjacent birth cohorts that were, or were not, exposed to extra vitamin D from margarine fortification prenatally only, but all unexposed thereafter, we assume that all potential confounders are equally distributed in both groups, and hence that control for confounding is not needed. Though we are unaware of such changes, we cannot exclude the possibility that other societal, environmental or behavioural changes coinciding with the change in fortification practice took place. However, changes in potential risk factors for paediatric fractures during the study period 1996-2012 may also be considered, such as secular trends in weight status, high-impact sports, better safety on playgrounds, onset of puberty, etc. Nonetheless, recent studies have found tendencies for a leveling-off of the trend in overweight and obesity among Danish adolescents (age range 11-16 years) during $2002-2010^{(38,39)}$. Similarly, the rate of injuries among children and youth occurring during home and leisure activities (as a proxy for physical activity), that is, at playgrounds and in sports participation, were stable during the period of interest ${ }^{(40)}$. An increasing number of Danish children are diagnosed with 
(a)

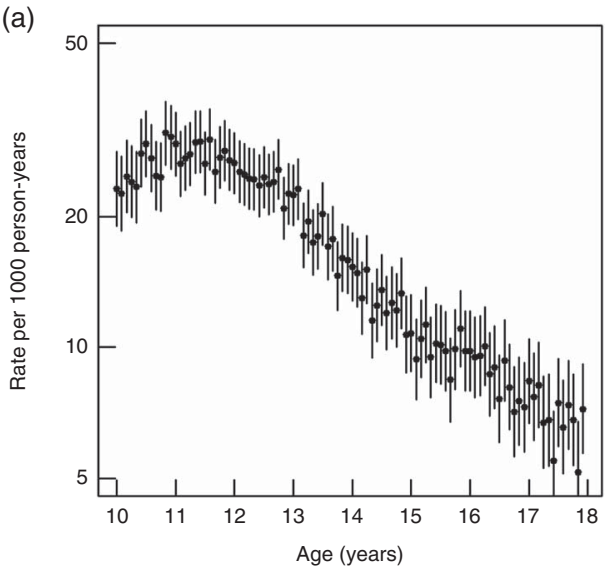

(c)

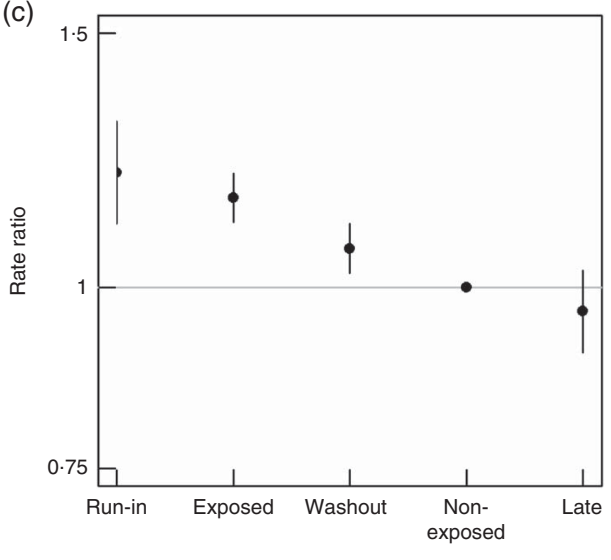

(b)

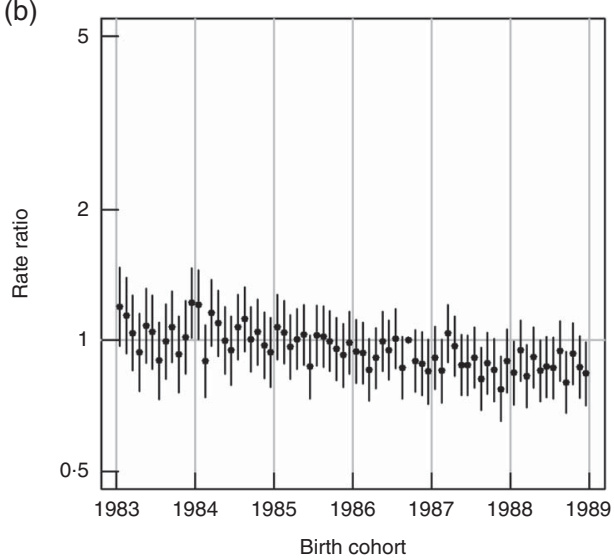

(d)

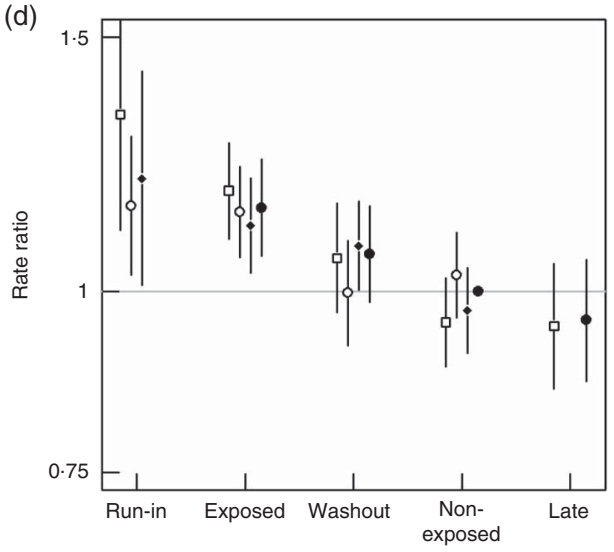

Birth cohort exposure group

Fig. 4. Age and birth cohort effects girls born in 1983-1988. (a) Age specific fracture rates per 1000 person-years and $95 \% \mathrm{Cl}$ for girls born in September 1986 , (b) rate ratio relative to September 1986 cohort, (c) rate ratios by birth cohort exposure groups ('Non-exposed' cohort is reference). and (d) rate ratios by birth cohort exposure group and season of birth (birth season 'August-October' and 'Non-exposed' cohort is reference). $\square$, November-January; $\bigcirc$, February-April; $\$$, May-July; , August-October.

precocious puberty from the mid-1990s and onwards ${ }^{(41)}$, although very few cases in total have been registered in the period 1993-2001 $\left(n\right.$ 670) ${ }^{(42)}$. Also, other Danish studies have suggested a decline in the age of onset of puberty, among both girls and boys, from 1991 to $2008^{(43,44)}$. However, the decline was about 3-4 months over a 15 -year period, and thus considered minor with regard to the adjacent birth cohorts that we studied over a maximum span of 5 years. Hence, the unchanging fluctuations in the trend of these major risk factors of fractures cannot explain the decreasing drift seen in our data.

The decreasing fracture trend may potentially be explained by the marked decrease in bicycle and pedestrian accidents related to road traffic injuries during 1990-2009 ${ }^{(40)}$, especially as fractures of the shoulders, arms, hands and wrist were those represented in our data set, and are the body parts most likely to be injured in bicycle accidents ${ }^{(40)}$. Also, the seasonal pattern in bicycle accidents, with particularly high rates during spring and summer compared with winter and autumn, was similar between the fracture rate pattern in our study and that in other studies ${ }^{(45-52)}$. Climatically, Denmark has a large seasonal variation in daylight length, temperature and precipitation. This seasonal variation might also contribute to periodic changes in outdoor physical activities among children during the year ${ }^{(53,54)}$.
The relationship between vitamin D status during fetal life and long-term bone health has not previously been widely examined, and results from earlier observational studies showed either no association or a direct association. In the Avon Longitudinal Study of Parents and Children (ALSPAC) study, UV-B-exposure during the third trimester of pregnancy was used as a proxy for vitamin D status, and the exposure was directly associated with bone mineral content (BMC), bone area and BMD among 6955 children at age 9.9 years $^{(8)}$. However, later, another report from the ALSPAC study showed no association with maternal $25(\mathrm{OH}) \mathrm{D}$ and total body BMC or spine BMC among 3960 offspring, after adjusting for offspring age by dual-energy X-ray absorptiometry scan ${ }^{(10)}$. Correspondingly, Danish Cohort studies showed no association between maternal $25(\mathrm{OH}) \mathrm{D}$ concentrations during pregnancy and fractures among the offspring at ages $0-18$ and $0-21$ years ${ }^{(12,37)}$. Findings from the Southampton Women Survey suggested that both $25(\mathrm{OH}) \mathrm{D}$ and estimated UV-B radiation during late pregnancy (mean gestation week of 34) were directly associated with bone-mineral accrual among 198 children up to age 9 years ${ }^{(10)}$. Finally, the Western Australian Pregnancy Cohort (Raine), which followed 341 offspring up to the age of 20 years, showed a direct association between maternal 25(OH)D measured 

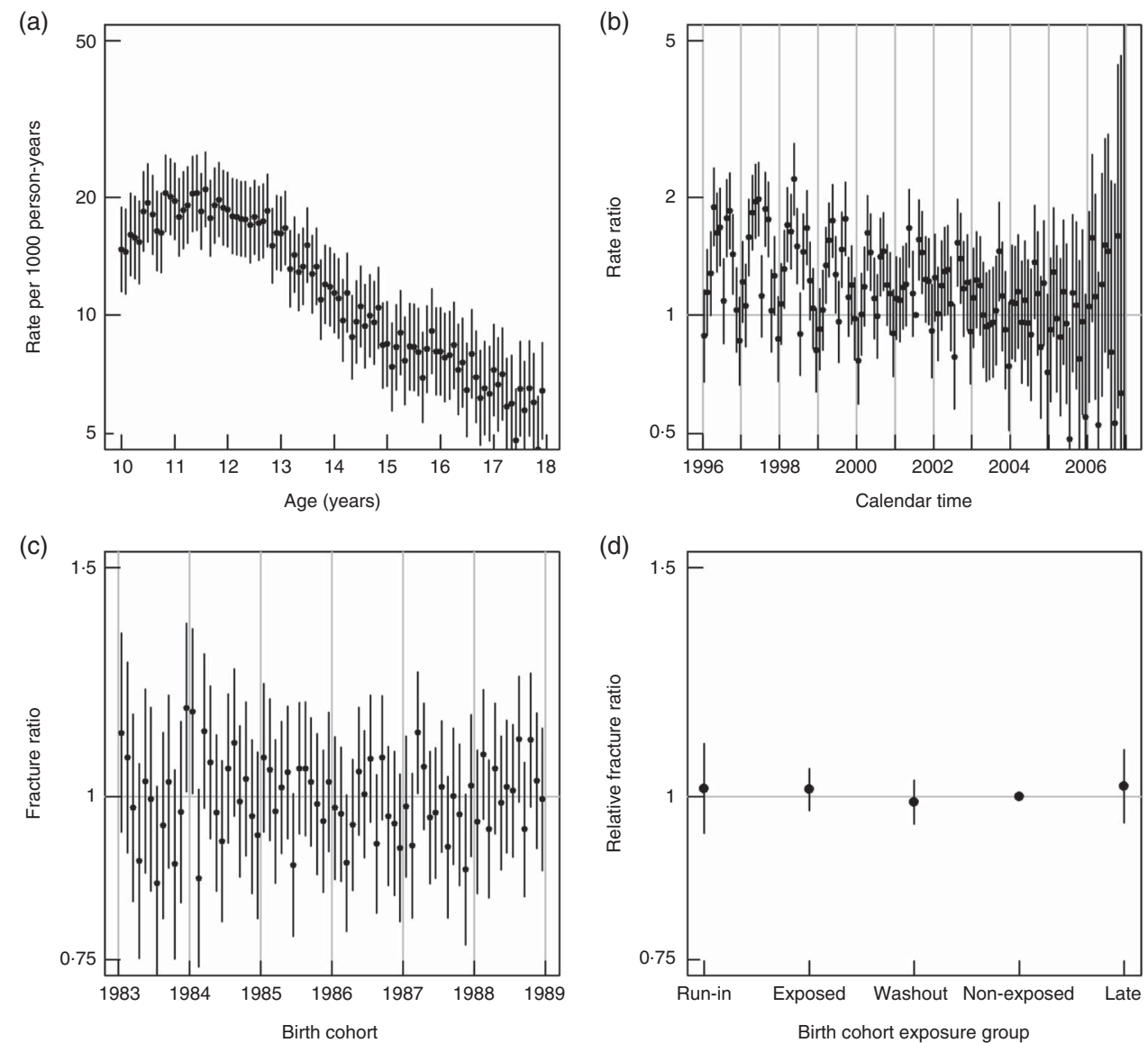

Fig. 5. Age and period effects for girls with fractures occurring from 1996-2007. (a) Age specific fracture rates per 1000 person-years and $95 \% \mathrm{Cl}$ for girls in June 2001, (b) rate relative to the July 2001 rate, (c) observed $v$. expected number of fractures conditional on the estimated age and period rates and (d) cohort effect by birth cohort exposure group relative to the cohort effect in the 'Non-exposed' cohort.

Table 2. Age-adjusted facture rate by month of fracture (July is the reference)

(Rate ratios (RR) and $95 \%$ confidence intervals)

\begin{tabular}{lccccc}
\hline & \multicolumn{2}{c}{ Boys } & & \multicolumn{2}{c}{ Girls } \\
\cline { 2 - 3 } \cline { 5 - 6 } Month of fracture & $\mathrm{RR}$ & $95 \% \mathrm{Cl}$ & & $\mathrm{RR}$ & $95 \% \mathrm{Cl}$ \\
\hline January & 0.92 & $0.86,0.98$ & & 1.04 & $0.96,1.13$ \\
February & 1.04 & $0.97,1.11$ & & 1.14 & $1.05,1.24$ \\
March & 1.21 & $1.13,1.28$ & & 1.37 & $1.27,1.48$ \\
April & 1.48 & $1.40,1.57$ & & 1.55 & $1.43,1.67$ \\
May & 1.67 & $1.58,1.77$ & & 1.71 & $1.58,1.84$ \\
June & 1.44 & $1.36,1.53$ & & 1.40 & $1.29,1.51$ \\
July & & 1 & & 1 \\
August & 1.73 & $1.63,1.83$ & & 1.57 & $1.45,1.70$ \\
September & 1.48 & $1.40,1.57$ & & 1.65 & $1.53,1.78$ \\
October & 1.02 & $0.96,1.09$ & & 1.20 & $1.11,1.31$ \\
November & 0.93 & $0.87,1.00$ & & 1.17 & $1.08,1.27$ \\
December & 0.70 & $0.65,0.76$ & & 0.90 & $0.82,0.98$ \\
\hline
\end{tabular}

between 16 and 20 weeks of gestation and total body BMC and $\mathrm{BMD}$ at age 20 years $^{(11)}$. The diverse results from these studies potentially relates to differences in sample sizes, or the covariates adjusted for, and the number and timing of serum $25(\mathrm{OH}) \mathrm{D}$ concentration measurement during pregnancy. No previous studies have examined the effect of prenatal food fortification.
Intervention studies with supplementation of vitamin $\mathrm{D}$ in pregnancy are currently ongoing ${ }^{(9,55)}$. One of them, the Maternal Vitamin D Osteoporosis Study (MAVIDOS), recently reported that maternal supplementation with 1000IU $(25 \mu \mathrm{g}) / \mathrm{d}$ cholecalciferol during pregnancy increased bone mass in winter-born infants only ${ }^{(56)}$; however, it could be more than a decade before the first bone fracture outcome data in children are available to properly formulate health policies.

A number of methodological issues deserve attention. From systematic review and meta-analysis of randomised controlled trials, there is evidence that vitamin D-fortified foods generally improve vitamin D status among both children and adults in a dose-dependent manner ${ }^{(57-59)}$. The Danish margarine fortification programme has been shown to account for approximately $0.36-0.57 \mu \mathrm{g}$ vitamin $\mathrm{D} /$ person per $\mathrm{d}^{(60)}$. These estimates are based on food disappearance data (food availability for human use), where the average purchasing of margarine was estimated to be 16000-17000 g margarine/person per year during the period $1983-1988^{(33)}$, which is equal to $44-46 \mathrm{~g}$ margarine/ person per $\mathrm{d}$, and the dose-equivalents for vitamin $\mathrm{D}$ from fortified margarine is on average $0.55-0.57 \mu \mathrm{g} /$ person per $\mathrm{d}^{(33)}$. Furthermore, from the Danish dietary habit survey from 1985 , the median vitamin D intake was $2 \cdot 8 \mu \mathrm{g} / \mathrm{d}$ among women aged $23-50$ years $^{(61)}$. Margarine consumption contributed to $13 \%$ of 
this vitamin $\mathrm{D}$ intake, which is equivalent to $0.36 \mu \mathrm{g}^{(61)}$. It is possible that this amount was too small to influence fracture risk at the population level, even when we confined the analysis to those pregnancies with habitually low vitamin $\mathrm{D}$ during late trimesters, that is, those giving birth during winter months. Unfortunately, we lack information on vitamin D status among Danish women in the reproductive age during the fortification period, but for comparision, a study showed that among 850 pregnant women recruited from the second-largest city in Denmark in 1988-1989 (the period without fortification), only 6.3 of the pregnant women had a serum $25(\mathrm{OH}) \mathrm{D}$ concentration $\leq 25 \mathrm{nmol} / \mathrm{l}^{(12)}$; the median concentrations of $76.2(95 \% \mathrm{CI}$ $23 \cdot 0,152 \cdot 1) \mathrm{nmol} / \mathrm{l}$ were also above the 'optimal level' as stated by the US Endocrine Society ${ }^{(62,63)}$. For replication, the Finish vitamin D fortification programme (initiated in 2003) may be an option because Finland, like Denmark, has access to nationwide individual registration of fractures ${ }^{(64)}$.

There are also limitations to the statistical model. The ageperiod analysis assumed that the overall trend in the fractures rates could be attributed to a period effect. Bearing in mind the identifiability problem between age, period and cohort, the data cannot inform whether the age-period approach is more appropriate than the age-cohort analysis. To help elucidate the 'true' period effect, we suggest, at this point, a National trend analysis of paediatric fractures for birth cohorts from several decades and not only births from 1983 to 1988.

The strength of the present study lies with the use of comprehensive registers covering the entire Danish population. This enables us to capture all fracture information in the study population from the NPR, which is a high-quality national mandatory registration system initiated in 1977 . The accuracy of the NPR has not been formally assessed as regards paediatric bone ICD coding. Fracture diagnosis coding has high precision in adults ${ }^{(65)}$, and treatment of childhood fractures takes place at the same hospital units as treatment of fractures in adulthood, with very little extent of fracture treatment in general practice.

\section{Conclusion}

The study did not provide evidence that prenatal exposure to extra vitamin $\mathrm{D}$ from a mandatory fortification programme, adding1.25 $\mu \mathrm{g}$ vitamin $\mathrm{D} / 100 \mathrm{~g}$ margarine, was sufficient to influence the risk of fractures in late childhood, regardless of season of birth. Replication studies are needed. There was a decreasing trend in fracture events occurring in the birth cohort of 1983-1988, which might be explained by secular trends of bicycle accidents in the period, rather than by differences in the birth cohorts.

\section{Supplementary material}

For supplementary material/s referred to in this article, please visit https://doi.org/10.1017/S000711451700071X

\section{Acknowledgements}

This study was supported by the Danish Council for Strategic Research (11-116213); and the University of Southern Denmark. The sources of funding had no influence on the manuscript.
B. L. H. conceived and designed the D-tect observational study. M. N. H., B. A. and B. L. H. conceived this sub-study. M. N. H., P. F. and C. O. performed the statistical analysis. M. N. H., P. F., B. A. and B. L. H. wrote the paper with contributions from all authors. M. N. H. has primary responsibility for the final content. All authors have read and approved the final manuscript.

C. C. has received consultancy, lecture fees and honoraria from Alliance for Better Bone Health, Amgen, Eli Lilly, GSK, Medtronic, Merck, Novartis, Pfizer, Roche, Servier, Takeda and Union Chimique Belge (UCB). B. A. conducts epidemiological studies through research contracts between his institution and Novartis and UCB Pharma. All other authors have nothing to disclose.

\section{References}

1. Woolcott CG, Giguere Y, Weiler HA, et al. (2016) Determinants of vitamin D status in pregnant women and neonates. Can J Public Health 107, e410-e416.

2. Andersen R, Brot C, Jakobsen J, et al. (2013) Seasonal changes in vitamin D status among Danish adolescent girls and elderly women: the influence of sun exposure and vitamin D intake. Eur J Clin Nutr 67, 270-274.

3. Ministry of Food (1984) Executive order on margarine no. 196 20 May 1985. Act on margarine no. 189. Comments on the bill.

4. Randsborg PH, Gulbrandsen P, Saltyte BJ, et al. (2013) Fractures in children: epidemiology and activity-specific fracture rates. J Bone Joint Surg Am 95, e42.

5. Anderson PH, Atkins GJ, Turner AG, et al. (2011) Vitamin D metabolism within bone cells: effects on bone structure and strength. Mol Cell Endocrinol 347, 42-47.

6. Galthen-Sorensen M, Andersen LB, Sperling L, et al. (2014) Maternal 25-hydroxyvitamin D level and fetal bone growth assessed by ultrasound: a systematic review. Ultrasound Obstet Gynecol 44, 633-640.

7. Javaid MK, Crozier SR, Harvey NC, et al. (2006) Maternal vitamin $\mathrm{D}$ status during pregnancy and childhood bone mass at age 9 years: a longitudinal study. Lancet $\mathbf{3 6 7}$, $36-43$.

8. Sayers A \& Tobias JH (2009) Estimated maternal ultraviolet B exposure levels in pregnancy influence skeletal development of the child. J Clin Endocrinol Metab 94, 765-771.

9. Viljakainen HT, Korhonen T, Hytinantti T, et al. (2011) Maternal vitamin D status affects bone growth in early childhood a prospective cohort study. Osteoporos Int 22, 883-891.

10. Lawlor DA, Wills AK, Fraser A, et al. (2013) Association of maternal vitamin D status during pregnancy with bonemineral content in offspring: a prospective cohort study. Lancet 381, 2176-2183.

11. Zhu K, Whitehouse AJ, Hart P, et al. (2014) Maternal vitamin D status during pregnancy and bone mass in offspring at 20 years of age: a prospective cohort study. J Bone Miner Res 29, 1088-1095.

12. Petersen SB, Olsen SF, Molgaard C, et al. (2014) Maternal vitamin $\mathrm{D}$ status and offspring bone fractures: prospective study over two decades in Aarhus City, Denmark. PLOS ONE 9, e114334.

13. Clark EM, Tobias JH \& Ness AR (2006) Association between bone density and fractures in children: a systematic review and meta-analysis. Pediatrics 117, e291-e297.

14. Manias K, McCabe D \& Bishop N (2006) Fractures and recurrent fractures in children; varying effects of environmental factors as well as bone size and mass. Bone 39, 652-657. 
15. Mayranpaa MK, Viljakainen HT, et al. (2012) Impaired bone health and asymptomatic vertebral compressions in fractureprone children: a case-control study. J Bone Miner Res 27, 1413-1424.

16. Olney RC, Mazur JM, Pike LM, et al. (2008) Healthy children with frequent fractures: how much evaluation is needed? Pediatrics 121, 890-897.

17. Clark EM, Ness AR, Bishop NJ, et al. (2006) Association between bone mass and fractures in children: a prospective cohort study. J Bone Miner Res 21, 1489-1495.

18. Ferrari SL, Chevalley T, Bonjour JP, et al. (2006) Childhood fractures are associated with decreased bone mass gain during puberty: an early marker of persistent bone fragility? J Bone Miner Res 21, 501-507.

19. Flynn J, Foley S \& Jones G (2007) Can BMD assessed by DXA at age 8 predict fracture risk in boys and girls during puberty?: an eight-year prospective study. J Bone Miner Res 22, 1463-1467.

20. Cheng S, Xu L, Nicholson PH, et al. (2009) Low volumetric BMD is linked to upper-limb fracture in pubertal girls and persists into adulthood: a seven-year cohort study. Bone 45, 480-486.

21. Maasalu K, Raukas M \& Märtson A (2009) Children's fractures in Estonia: population based study. Bone 45, S59-S111.

22. Hedstrom EM, Svensson O, Bergstrom U, et al. (2010) Epidemiology of fractures in children and adolescents. Acta Orthop 81, 148-153.

23. Baker R, Orton E, Tata LJ, et al. (2016) Epidemiology of poisonings, fractures and burns among 0-24 year olds in England using linked health and mortality data. Eur J Public Health 26, 940-946.

24. Mayranpaa MK, Makitie O \& Kallio PE (2010) Decreasing incidence and changing pattern of childhood fractures: a population-based study. J Bone Miner Res 25, 2752-2759.

25. Cooper C, Dennison EM, Leufkens HG, et al. (2004) Epidemiology of childhood fractures in Britain: a study using the general practice research database. J Bone Miner Res 19, 1976-1981.

26. Moon RJ, Harvey NC, Curtis EM, et al. (2016) Ethnic and geographic variations in the epidemiology of childhood fractures in the United Kingdom. Bone 85, 9-14.

27. Lyons RA, Delahunty AM, Kraus D, et al. (1999) Children's fractures: a population based study. Inj Prev 5, 129-132.

28. Lyons RA, Sellstrom E, Delahunty AM, et al. (2000) Incidence and cause of fractures in European districts. Arch Dis Child 82, 452-455.

29. Jacobsen R, Abrahamsen B, Bauerek M, et al. (2013) The influence of early exposure to vitamin D for development of diseases later in life. BMC Public Health 13, 515.

30. Craig P, Cooper C, Gunnell D, et al. (2012) Using natural experiments to evaluate population health interventions: new Medical Research Council guidance. J Epidemiol Community Health 66, 1182-1186.

31. Haradsdóttir J \& Thaarup S (1989) Tilscetning af vitaminer og mineraler til levnedsmidler (The Fortification of Foods With Vitamins and Minerals). Report, no. 45. Copenhagen: Nordic Council of Ministers

32. Jensen CB, Stougård M, Sørensen TI, et al. (2016) Does prenatal exposure to vitamin D-fortified margarine and milk alter birth weight? A societal experiment - CORRIGENDUM. Br J Nutr 116, 377-379.

33. Fagt S \& Trolle E (2001) Forsyning af fødevarer 1955-1999. Udvikling $i$ danskernes kost - forbrug, indkøb og vaner (The Supply of Food from 1955 to 1999. Overview of the Developments in the Danish Diet - Consumption, Purchasing and Habits). Søborg: Danish Veterinary and Food Administration.

34. Nickelsen TN (2001) Data validity and coverage in the Danish National Health Registry. A literature review. Ugeskr Laeger 164, 33-37.
35. Lynge E, Sandegaard JL \& Rebolj M (2011) The Danish National Patient Register. Scand J Public Health 39, 7 Suppl., 30-33.

36. Carstensen B (2016) Age-period-cohort models for the Lexis diagram. Br J Nutr 116, 377-379.

37. Petersen SB, Strom M, Maslova E, et al. (2015) Predicted vitamin $\mathrm{D}$ status during pregnancy in relation to offspring forearm fractures in childhood: a study from the Danish National Birth Cohort. Br J Nutr 114, 1900-1908.

38. Pearson S, Hansen B, Sorensen TI, et al. (2010) Overweight and obesity trends in Copenhagen schoolchildren from 2002 to 2007. Acta Paediatr 99, 1675-1678.

39. Schmidt MC, Rokholm B, Sjoberg BC, et al. (2013) Trends in prevalence of overweight and obesity in Danish infants, children and adolescents - are we still on a plateau? PLOS ONE 8, e69860.

40. Møller H, Damm M \& Laursen B (2012) Ulykker i Danmark 1990-2009 (Accidents in Denmark 1990-2009). Copenhagen: National Institute of Public Health, University of Southern Denmark.

41. Mogensen SS, Aksglaede L, Mouritsen A, et al. (2011) Diagnostic work-up of 449 consecutive girls who were referred to be evaluated for precocious puberty. J Clin Endocrinol Metab 96, 1393-1401.

42. Teilmann G, Pedersen CB, Jensen TK, et al. (2005) Prevalence and incidence of precocious pubertal development in Denmark: an epidemiologic study based on national registries. Pediatrics 116, 1323-1328.

43. Aksglaede L, Sorensen K, Petersen JH, et al. (2009) Recent decline in age at breast development: the Copenhagen Puberty Study. Pediatrics 123, e932-e939.

44. Sorensen K, Aksglaede L, Petersen JH, et al. (2010) Recent changes in pubertal timing in healthy Danish boys: associations with body mass index. J Clin Endocrinol Metab 95, 263-270.

45. Sinikumpu JJ, Pokka T \& Serlo W (2013) The changing pattern of pediatric both-bone forearm shaft fractures among 86,000 children from 1997 to 2009. Eur J Pediatr Surg 23, 289-296.

46. Bell SW, McLaughlin D \& Huntley JS (2012) Paediatric forearm fractures in the west of Scotland. Scott Med J 57, 139-143.

47. Heideken J, Svensson T, Blomqvist P, et al. (2011) Incidence and trends in femur shaft fractures in Swedish children between 1987 and 2005. J Pediatr Orthop 31, 512-519.

48. Ryan LM, Teach SJ, Searcy K, et al. (2010) Epidemiology of pediatric forearm fractures in Washington, DC. J Trauma 69 , 4 Suppl., S200-S205.

49. Kalenderer O, Gurcu T, Reisoglu A, et al. (2006) The frequency and distribution of fractures in children presenting to the emergency service. Acta Orthop Traumatol Turc 40, 384-387.

50. Lautman S, Bergerault F, Bonnard C, et al. (2003) Epidemiological survey of wrist fractures in children. Rev Chir Orthop Reparatrice Appar Mot 89, 399-403.

51. Ljungberg E, Rosberg HE \& Dahlin LB (2003) Hand injuries in young children. J Hand Surg Br 28, 376-380.

52. Wareham K, Johansen A, Stone MD, et al. (2003) Seasonal variation in the incidence of wrist and forearm fractures, and its consequences. Injury 34, 219-222.

53. Kolle E, Steene-Johannessen J, Andersen LB, et al. (2009) Seasonal variation in objectively assessed physical activity among children and adolescents in Norway: a crosssectional study. Int J Behav Nutr Phys Act 6, 36.

54. Kristensen PL, Korsholm L, Moller NC, et al. (2008) Sources of variation in habitual physical activity of children and adolescents: the European youth heart study. Scand J Med Sci Sports 18, 298-308. 
55. Harvey NC, Javaid K, Bishop N, et al. (2012) MAVIDOS Maternal Vitamin D Osteoporosis Study: study protocol for a randomized controlled trial. The MAVIDOS Study Group. Trials 13, 13.

56. Cooper C, Harvey NC, Bishop NJ, et al. (2016) Maternal gestational vitamin $\mathrm{D}$ supplementation and offspring bone health (MAVIDOS): a multicentre, double-blind, randomised placebo-controlled trial. Lancet Diabetes Endocrinol 4, 393-402.

57. Black LJ, Seamans KM, Cashman KD, et al. (2012) An updated systematic review and meta-analysis of the efficacy of vitamin D food fortification. J Nutr 142, 1102-1108.

58. Hennessy Á, Browne F, Kiely M, et al. (2017) The role of fortified foods and nutritional supplements in increasing vitamin D intake in Irish preschool children. Eur J Nutr 56, 1219-1231.

59. Piirainen T, Laitinen K \& Isolauri E (2007) Impact of national fortification of fluid milks and margarines with vitamin $D$ on dietary intake and serum 25-hydroxyvitamin D concentration in 4-year-old children. Eur J Clin Nutr 61, 123-128.
60. Jacobsen R, Hypponen E, Sorensen TI, et al. (2015) Gestational and early infancy exposure to margarine fortified with vitamin D through a National Danish Programme and the risk of type 1 diabetes: The D-Tect Study. PLOS ONE 10, e0128631.

61. Haraldsdóttir J, Holm L, Højmark Jensen J, et al. (1986) Dietary Habits in Denmark 1985, Volume 1. Main Results. Søborg: National Food Agency (Levnedsmiddelstyrelsen).

62. Holick MF, Binkley NC, Bischoff-Ferrari HA, et al. (2011) Evaluation, treatment, and prevention of vitamin D deficiency: an Endocrine Society clinical practice guideline. J Clin Endocrinol Metab 96, 1911-1930.

63. Rosen CJ, Abrams SA, Aloia JF, et al. (2012) IOM committee members respond to Endocrine Society vitamin D guideline. J Clin Endocrinol Metab 97, 1146-1152.

64. Pietinen P, Mannisto S, Valsta LM, et al. (2010) Nutrition policy in Finland. Public Health Nutr 13, 901-906.

65. Vestergaard P \& Mosekilde L (2002) Fracture risk in patients with celiac disease, Crohn's disease, and ulcerative colitis: a nationwide follow-up study of 16,416 patients in Denmark. Am J Epidemiol 156, 1-10. 